EPJ manuscript No.

(will be inserted by the editor)

\title{
Nucleon Structure from Lattice QCD
}

\author{
David Richards \\ Jefferson Laboratory, 12000 Jefferson Avenue, Newport News, VA 23606, USA
}

November 12, 2007

\begin{abstract}
Recent advances in lattice field theory, in computer technology and in chiral perturbation theory have enabled lattice QCD to emerge as a powerful quantitative tool in understanding hadron structure. I describe recent progress in the computation of the nucleon form factors and moments of parton distribution functions, before proceeding to describe lattice studies of the Generalized Parton Distributions (GPDs). In particular, I show how lattice studies of GPDs contribute to building a three-dimensional picture of the proton, I conclude by describing the prospects for studying the structure of resonances from lattice QCD.
\end{abstract}

PACS. 12.38.Gc Lattice QCD calculations - 13.40.Gp Electromagnetic form factors - 13.60.Fz Elastic and Compton scattering

\section{Introduction}

The ability for lattice QCD to make increasingly precise, ab initio calculations of hadron structure has been driven by advances in three areas: algorithmic advances, enabling both chiral symmetry to be more faithfully represented on the lattice and gauge configurations to be generated more efficiently, computational advances represented by the availability of special-purpose and leadership-class computers and by commodity clusters, and theoretical advances enabling extrapolations to be performed from the quark masses employed in the computation to the physical up- and down-quark masses. In this talk, I will show the impact of these advances on hadron structure, and provide a roadmap for future calculations. The layout of the rest of this talk is as follows. In the next section, I will describe how hadron-structure calculations proceed in lattice QCD. I will then review benchmark calculations, such as that of the nucleon's electromagnetic form factors. Section 4 will describe calculations of moments of Generalized Parton Discributions (GPDs) and the impact such calculations are having on our understanding of hadron structure. I will conclude with prospects for future calculations.

\section{Anatomy of a nucleon-structure calculation}

Hadron structure is expressed through quantities as the electromagnetic form factors, describing the distribution of charge and currents within a hadron, and the polarized and unpolarized parton structure functions, describing how the longitudinal momentum fraction and spin is apportioned amongst the constituents. Recently, Generalized Parton Distributions have been introduced[1-3], encompassing both of the concepts above, but allowing a three-dimensional picture of the nucleon to be constructed.
GPDs are expressed as the matrix elements of lightcone correlation functions $\mathcal{O}_{\Gamma}(x)$ :

$$
\mathcal{O}_{\Gamma}(x)=\int \frac{d \lambda}{4 \pi} e^{i \lambda x} \bar{q}\left(\frac{-\lambda n}{2}\right) \Gamma \mathcal{P} e^{-i g \int_{-\lambda / 2}^{\lambda / 2} d \alpha n \cdot A(\alpha n)} q\left(\frac{\lambda}{2}\right)
$$

where $n$ is a light-cone vector, the parallel-transporter is necessary to ensure gauge invariance, and the flavour indices on the quarks are suppressed. The familiar quark polarized and unpolarized parton structure functions are then forward matrix elements of this operator, with $\Gamma=$ $\gamma_{\mu}$ or $\gamma_{\mu} \gamma_{5}$ for the unpolarized and polarized distributions, respectively; the GPDs correspond to matrix elements in the off-forward direction, with different momenta for the incoming and outgoing partons.

The use of a Euclidean lattice precludes the measurement of these matrix elements directly. Instead with appeal to the Operator Product Expansion to expand the operator $\mathcal{O}(x)$ about the light cone, yielding a set of local operators which can be measured on a Euclidean lattice, and furthermore analytically continued to Minkowski space. In particular, the moments, with respect to Bjorken- $x$, of the quark structure functions are obtained in terms of the forward matrix elements

$$
\mathcal{O}_{\Gamma}^{\left\{\mu_{1} \ldots \mu_{n}\right\}}=\bar{q}\left(\gamma_{5}\right) \gamma^{\left\{\mu_{1}\right.} i D^{\mu_{2}} \ldots D^{\left.\mu_{n}\right\}} q .
$$

Specifically, for the unpolarized distribution for a nucleon carrying momentum $\boldsymbol{p}$, we have

$$
\left\langle\boldsymbol{p}\left|\mathcal{O}_{q}^{\mu_{1} \ldots \mu_{n}}\right| \boldsymbol{p}\right\rangle \longrightarrow \int_{0}^{1} d x x^{n-1} q(x),
$$

where I have suppressed the Lorentz structure.

We have seen in the talk of Christof Gattringer how the spectrum is obtained in lattice QCD through the measurement of so-called two-point functions representing the 
Euclidean correlators of two operators that interpolate between a state and the vacuum. Nucleon structure is investigated through the measurement of three-point functions, illustrated in Figure 1, representing the Euclidean correlation functions of three operators:

$C_{3 \mathrm{pt}}\left(t_{\mathrm{snk}}, \tau ; \boldsymbol{p}, \boldsymbol{q}\right)=\sum_{\boldsymbol{x}, \boldsymbol{y}} e^{-i \boldsymbol{p} \cdot \boldsymbol{x}-i \boldsymbol{q} \cdot \boldsymbol{y}}\left\langle 0\left|J\left(\boldsymbol{x}, t_{\mathrm{snk}}\right) \mathcal{O}(\boldsymbol{y}, \tau) J^{\dagger}(0)\right| 0\right\rangle$

where $J$ is an operator that interpolates between the state and the vacuum. Note that the disconnected contribution in the right-hand panel of Figure 1 is far more computationally demanding that the connected piece; for most of the following, I will emphasise the calculation of flavour non-singlet, or isovector, quantities, for which the disconnected piece vanishes.

Following [4], we insert complete sets of states between the operators in eqn. 4; the time-slice sum projects onto states of definite momentum, and we obtain:

$$
\begin{aligned}
& C_{3 \mathrm{pt}}\left(t_{\mathrm{snk}}, \tau ; \boldsymbol{p}, \boldsymbol{q}\right)=\sum_{n=1, \ldots} e^{-E_{n}(\boldsymbol{p})\left(t_{\mathrm{snk}}-\tau\right)} e^{-E_{n}(\boldsymbol{p}+\boldsymbol{q}) \tau} \times \\
& \langle 0|J(0)| n, \boldsymbol{p}\rangle\langle n, \boldsymbol{p}|\mathcal{O}(0)| n, \boldsymbol{p}+\boldsymbol{q}\rangle\left\langle n, \boldsymbol{p}+\boldsymbol{q}\left|J^{\dagger}(0)\right| 0\right\rangle .(5)
\end{aligned}
$$

For sufficiently large $\tau, t_{\mathrm{snk}}-\tau, C_{3 \mathrm{pt}}$ is dominated by the lowest-lying state, and we can obtain $\langle n=1, \boldsymbol{p}|\mathcal{O}| n=1, \boldsymbol{p}\rangle$ after elimination of the vacuum-to-state matrix elements from the corresponding two-point functions.

Computations of hadron structure are being performed by several groups, using a variety of fermion and gaugefield discretisations. The common theme is calculations at increasingly light values of the pion mass, enabling lattice calculations, together with chiral effective theory, to provide insight into physics at the physical quark masses; recent reviews are contained in ref. $[5,6]$. During the remainder of this talk, I will in large part quote results from the LHP Collaboration. We employ a hybrid approach, using lattices generated using $2+1$ flavours of Asqtad quarks, corresponding to degenerate $u / d$ and strange, by the MILC Collaboration[7], but with domain-wall fermions (DWF) for the valence quarks, computed on HYP-smeared lattices[8]. The generation of lattices using Asqtad fermions is very computationally efficient, whilst the DWF valence quarks have the desired chiral-symmetry properties, avoiding the much operator mixing and simplifying the matching of our results to the continuum. Whilst this approach violates unitarity at finite lattice spacing, we expect this approach to yield the correct theory in the continuum limit[9]. In this talk, I will present results with pion masses down to around $360 \mathrm{MeV}$.

\section{Electromagnetic Form Factors}

These describe the distribution of charge and current within the nucleon, and remain the subject of intense experimental and theoretical interest. They are the most straightforward quantity to measure in a lattice calculation, corresponding to the matrix element of the electromagnetic

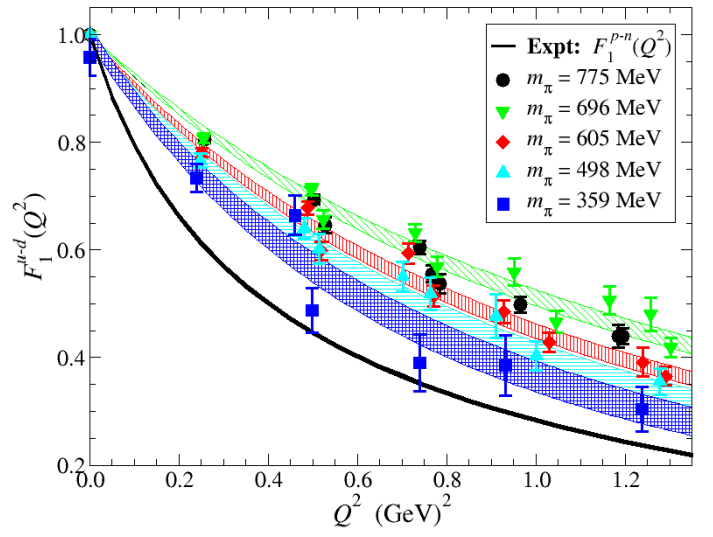

Fig. 2. The points show measurements of the isovector $F_{1}$ form factor at five values of the pion mass; the coloured bands are uncertainties from a pole-dominance fit to the data. The solid line is a parametrisation of the experimental data[11].

current $V_{\mu}=\bar{q} \gamma_{\mu} q$. Figure 2 shows the form factor $F_{1}\left(Q^{2}\right)$ determined by the LHP Collaboration[10], together with a parametrisation of the isovector experimental data[11]. The data show the approach to the experimental parametrisation at decreasing values of the pion mass used in the lattice calculation.

The slope of the form factor at $Q^{2}=0$ is related to the isovector charge radius $\left\langle r^{2}\right\rangle^{1 / 2}$. The left-hand panel in Figure 3 shows the charge radius obtained from the calculation above. A naive linear fit in $m_{\pi}^{2}$ would clear be far below the experimental points, illustrating the need to correctly describe the non-analytic behaviour in the approach to the physical light-quark masses. The curve shows the chiral extrapolation of the charge radius, using the finite-range regulator[12]. The right-hand panel shows the isovector mean square charge radius obtained by the QCDSF/UKQCD Collaborations, using two flavours of Wilson fermions, with pion masses down to around $350 \mathrm{MeV}$; the line is the chiral behaviour in the smallscale expansion.[13]

\section{Generalized Parton Distributions}

The electromagnetic form factors described above are particular cases of Generalized Parton Distributions. There are two GPDs corresponding to the choice $\Gamma=\gamma \cdot n$ in eqn. $1, H(x, \xi, t)$ and $E(x, \xi, t)$, and a further two corresponding to the $\Gamma=\gamma \cdot n \gamma_{5}$ in eqn. $1, \tilde{H}(x, \xi, t)$ and $\tilde{E}(x, \xi, t)$. The invariants are:

$$
\begin{aligned}
& t=-\Delta^{2}=-\left(P-P^{\prime}\right)^{2} \\
& \xi=-n \cdot \Delta / 2
\end{aligned}
$$

where $n_{\mu}$ is a light-cone vector, and $P^{\prime}, P$ are the fourmomenta of the incoming and outgoing states. Once again, we appeal to the operator product expansion to obtain the matrix elements of local operators, and both the form factors described above and the familiar parton distributions 


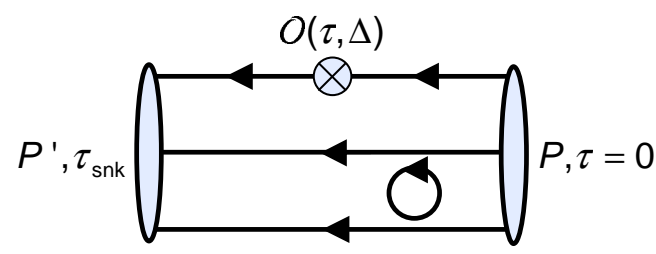

a)

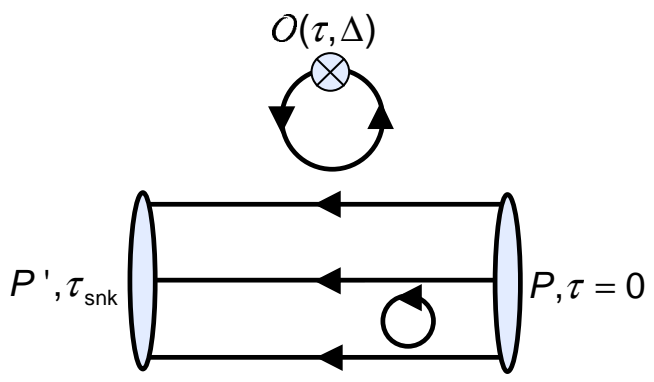

b)

Fig. 1. The left- and right-hand panels show the connected and disconnected contributions to the three-point function of eqn. 4.
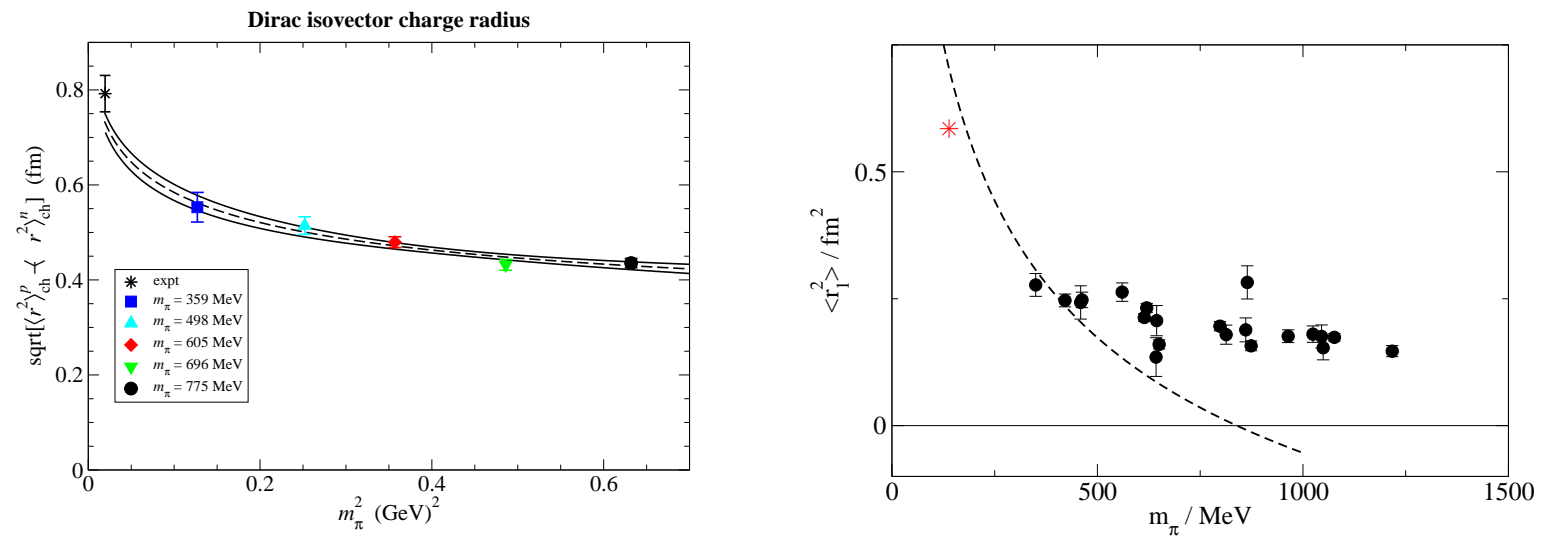

Fig. 3. The left-hand panel shows the isovector charge radius using a mixed Asqtad/DWF calculation[10], together with the chiral behavious using a finite-range regulator[12]. The right-hand panel shows the mean-square charge radius using two flavours of Wilson fermions[14], together with the chiral behaviour using the small-scale expansion[13].

emerge as special cases:

$$
\begin{aligned}
& H(x, 0,0)=q(x) \\
& \tilde{H}(x, 0,0)=\Delta q(x),
\end{aligned}
$$

and

$$
\int_{-1}^{1} d x H(x, \xi, t)=F_{1}(t)
$$

Note here that the $x$ runs from -1 to 1 , corresponding to antiquark and quark momentum fraction.

Before proceeding to discuss the new insights enabled by the study of GPDs, we begin by discussing the polarized and unpolarized distributions as a means of benchmarking our calculation. A fundamental measure of QCD is the nucleon's axial-vector charge $g_{A}$, corresponding to the choice $\Gamma=\gamma_{5}$; it has additional importance in its rôle as a fundamental low-energy constant of the theory that appears in the chiral expansion of other quantities. Figure 4 shows a calculation of the axial-vector charge by the LHP collaboration, together with the chiral extrapolation to the physical quark masses[15]. The consistency between the extrapolated lattice data and the experimental value is very encouraging, though the chiral behaviour for this quantity is very mild.

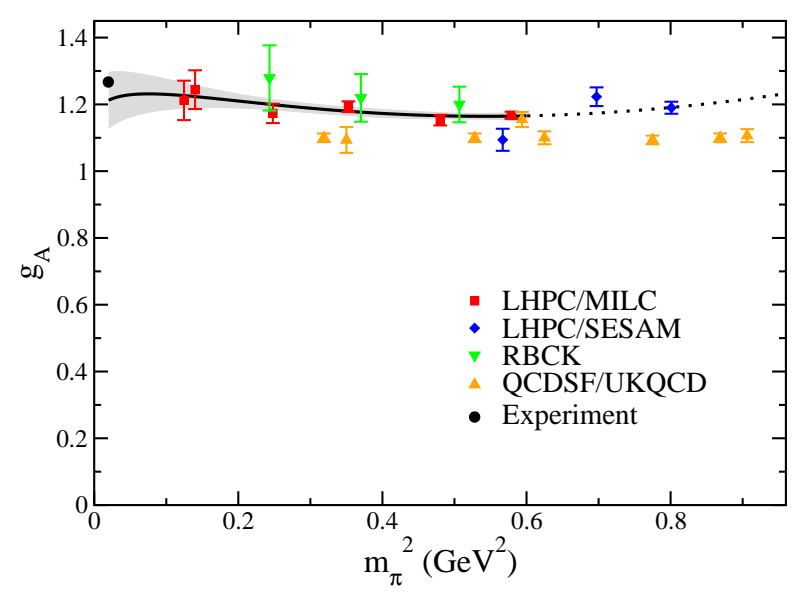

Fig. 4. The red squares show LHPC/MILC data for the axial-vector charge $g_{A}$; the two data points at the lowest pion mass correspond to to different volumes. The remaining lattice points are taken from QCDSF/UKQCD[16], RBCK[17] and SESAM[18]. 

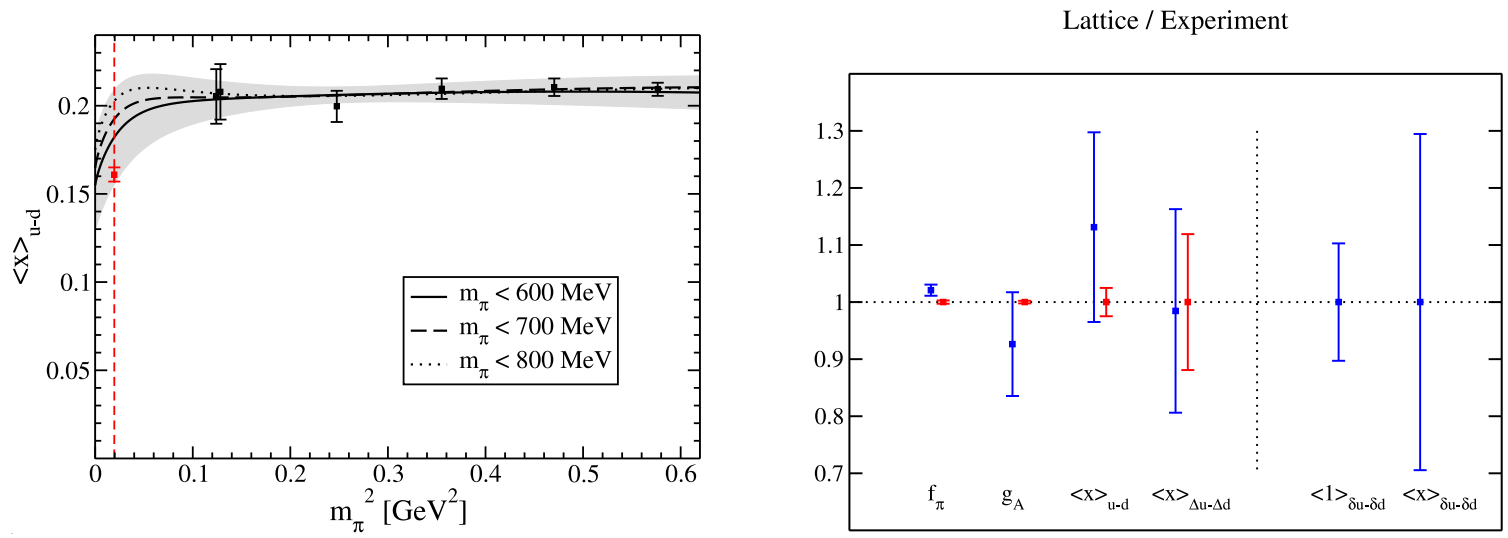

Fig. 5. The left-hand figure shows the momentum fraction carried by the valence quarks in a nucleon, together with the chiral extrapolation to the physical quark masses. The right-hand figure is a summary shows the lattice values at the physical pion masses for a range of benchmark quantities, normalized to the experimental value where known (blue)[19]; the errors on the corresponding experimental measurements are shown in red.

The non-analytic behaviour with decreasing quark mass is more strongly exhibited in the unpolarized distributions, and in particular in the calculation of the momentum fraction carried by the valence quarks in the nucleon. An analysis of the forward matrix elements for both the unpolarized and polarized distributions, as well as for the transversity, has been performed using a self-consistent chiral expansion[19]; data for the non-singlet momentum fraction, and a summary of the results for a range of benchmark quantities, is shown in Figure 5.

\subsection{New insights: origin of nucleon spin}

The excitement and signficance of GPDs arises from the new insights they can provide into hadron structure, spurrin new experimental initiatives such as the $12 \mathrm{GeV}$ upgrade at Jefferson Laboratory. There is thus a rigorous effort both by the LHP Collaboration in a hybrid DWF/Asqtad approach[20,19], and by the QCDSF/UKQCD Collaboration using two flavours of dynamical, improved Wilson quarks[21], to measure moments of GPDs.

An salient example of the new insights facilitated through the study of GPDs is how the spin of the nucleon is distributed amongst its constituents, and in particular the rôle of orbital angular momentum of the quarks. The spin carried by the quarks in a nucleon has long been the pursuit of lattice calculations, but no direct gauge-invariant definition of the orbital angular momentum appeared possible, though a new proposal was presented at this workshop[22]. However, it was realized that the total angular momentum carried by the quarks within a nucleon could be related to moments of GPDs through[23]

$$
J_{q}=\frac{1}{2} \int d x x(H(x, \xi, 0)+E(x, \xi, 0)) .
$$

A pioneering effort to measure the quark orbital angular momentum was performed in the quenched approximation to QCD[24]; Figure 6 shows more recent calculations by the LHPC and QCDSF/UKQCD Collaborations. Note that the calculations include only the connected pieces. None-the-less, they suggest that the total orbital angular momentum carried by the quarks within a nucleon is small, but that the orbital angular momentum carried by the individual quark flavours may be substantial.

Intimately related to the question of the origin of orbital angular momentum in the nucleon are the so-called transverse spin densities, corresponding to the choice $\Gamma=$ $\sigma^{\mu \nu} \gamma_{5}$ in eqn. 1. These can be studied in lattice QCD, complementing the experimental effort at investigating Tranverse-momentum-dependent PDFs. Figure 7 shows the lowest moment of the densities for an unpolarised quark in an transversely polarized nucleon, and for transversely polarized quarks in an unpolarized nucleon[27]. The right-hand plots reveal the strong distortion in the spin densities for a transversely polarized quark in an unpolarized nucleon, and are suggestive of a negative BoerMulders function[28].

\section{Structure of resonances}

I have emphasised in this talk the structure of the nucleon, and have so far said little of the structure of nucleon resonances. The one resonance that has been extensively studied in lattice QCD is the $\Delta$, and indeed the mass of the $\Delta$ is a benchmark calculation in the hadron spectrum. The $\Delta$ decays to $N \pi$ in a $P$-wave, and the finite spatial extent used in our lattices provides a momentum threshold forbidding such decays in most calculations at current volumes and pion masses. Nevertheless, extensive insight into the structure of QCD has been revealed, in particular through lattice calculations of $\Delta$ to $N$ electromagnetic transition form factors. In contrast to the electromagnetic form factors discussed earlier, these are sensitive to a quadrupole moment in the nucleon and hence deviations from spherical symmetry. Recently, there has been a calculation of the ratio of electric-to-magnetic (EMR) and Coulomb-to-magnetic (CMR) transition form factor ratios, shown in Figure 8, with sufficient accrucacy to ex- 

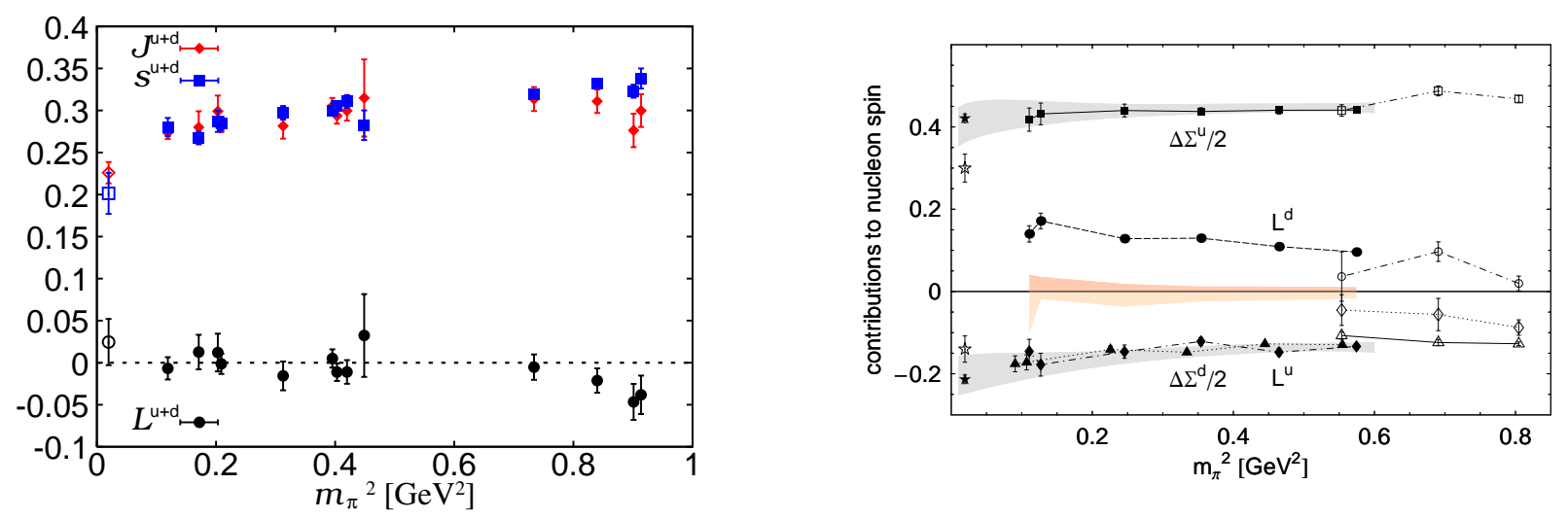

Fig. 6. The left-hand plot shows the spin carried by the quarks in the nucleon, the total angular momentum carried by the quarks, and the orbital angular momentum, in a calculation with two flavours of improved Wilson quarks[21]. The right-hand plot shows the decomposition of the spin between the quark flavours in a hybrid Asqtad/DWF approach[20], together with an experimental determination of the spin from HERMES[25,26]

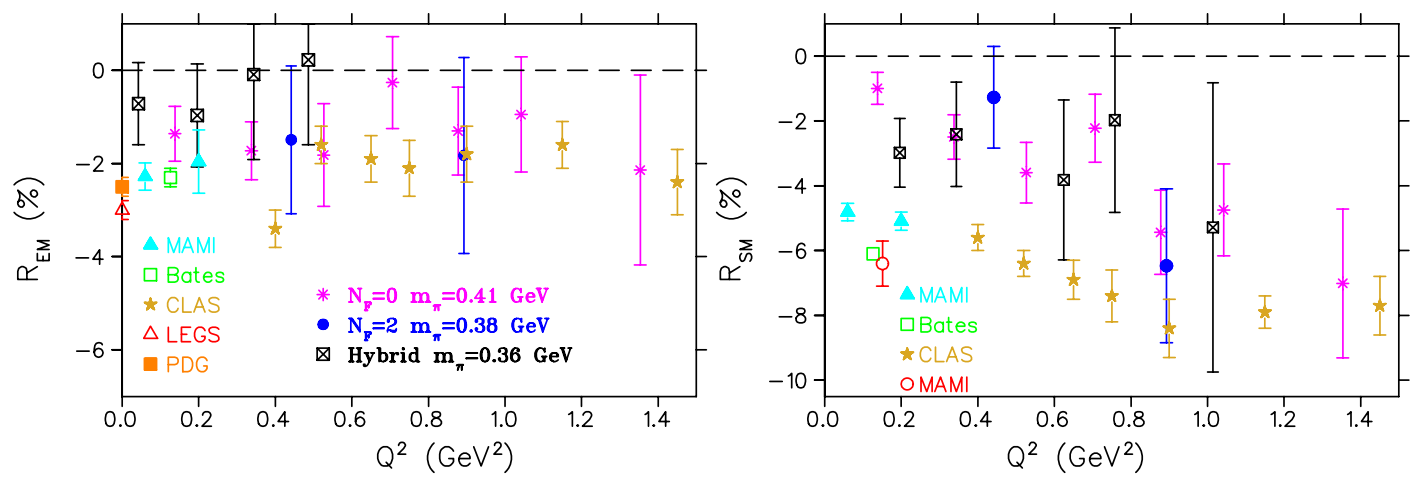

Fig. 8. The left- and right-hand plots show the ratios $\mathrm{EMR}=-\mathcal{G}_{E 2}\left(Q^{2}\right) / \mathcal{G}_{M 1}\left(Q^{2}\right) \quad$ and $\quad \mathrm{CMR} \quad=$ $-\left(|\boldsymbol{q}| / 2 m_{\Delta}\right) \mathcal{G}_{C 2}\left(q^{2}\right) / \mathcal{G}_{M 1}\left(q^{2}\right)[29]$, using both quenched and unquenched Wilson fermion actions, and using the Hybrid Asqtad/DWF approach. Also shown are experimental measurements.

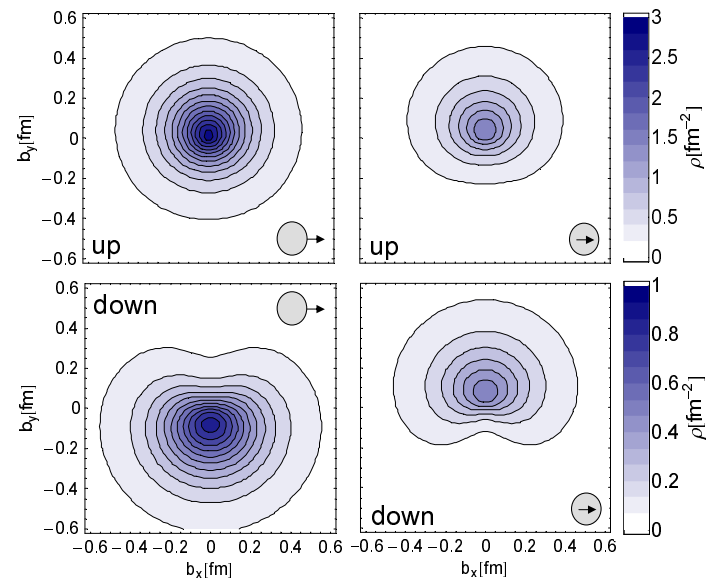

Fig. 7. Lowest moment of the densities of unpolarized quarks in a transversely polarized nucleon (left), and transversely polarized quarks in an unpolarized nucleon (right). The upper and lower figures correspond to the up and down quarks respectively. clude a zero value[29]; a non-zero value is indicative of deformations in the $N-\Delta$ system.

Given a sufficient basis of interpolating operators, the determination of the transitions to radial excitations will be feasible; we have heard in this workshop the success at extracting the electromagnetic properties of the Roper resonance from the CLAS data at Jefferson Laboratory[30]. Indeed, the first attempts at studying the Roper in lattice QCD are in progress[31].

\section{Conclusions and future prospects}

Theoretical and computational advances are enabling lattice QCD to have a vital impact on our understanding of hadron structure. Future challenges are two-fold: to proceed to values of the light-quark masses closer to the physical quark masses so to provide stronger constraints on the chiral extrapolations, and to delineate the contributions of the individual quark flavours. We heard in the talk of Christof Gattringer the successes at resolving the excited-state nucleon masses; the first attempts to study their properties should appear shortly. 
Acknowledgements: This work was supported by DOE contract DE-AC05-06OR23177 under which the Jefferson Science Associates, LLC operates the Thomas Jefferson National Accelerator Facility. I am grateful for collaboration with my colleagues in LHPC: D.B. Renner, J. Bratt, R.G. Edwards, M. Engelhardt, G. Fleming, Ph. Hägler, B. Musch, J.W. Negele, K. Orginos, A.V. Pochinsky, and W. Schroers

\section{References}

1. D. Mueller, D. Robaschik, B. Geyer, F.M. Dittes, J. Horejsi, Fortschr. Phys. 42, 101 (1994), hep-ph/9812448

2. X.D. Ji, Phys. Rev. D55, 7114 (1997), hep-ph/9609381

3. A.V. Radyushkin, Phys. Rev. D56, 5524 (1997), hep-ph/9704207

4. C. Gattringer, Excited hadrons on the lattice, in These proceedings (2007)

5. P. Hagler (2007), arXiv:0711.0819 [hep-lat]

6. K. Orginos, PoS LAT2006, 018 (2006)

7. C.W. Bernard et al., Phys. Rev. D64, 054506 (2001), hep-lat/0104002

8. A. Hasenfratz, F. Knechtli, Phys. Rev. D64, 034504 (2001), hep-lat/0103029

9. S.R. Sharpe, PoS LAT2006, 022 (2006), hep-lat/0610094

10. R.G. Edwards et al., PoS LAT2006, 121 (2006), hep-lat/0610007

11. J.J. Kelly, Phys. Rev. C70, 068202 (2004)

12. D.B. Leinweber, A.W. Thomas, R.D. Young, Phys. Rev. Lett. 86, 5011 (2001), hep-ph/0101211

13. M. Gockeler et al. (QCDSF), Phys. Rev. D71, 034508 (2005), hep-lat/0303019

14. M. Gockeler et al. (QCDSF/UKQCD), PoS. LATTICE2007 (2007), arXiv:0710.2159 [hep-lat]

15. R.G. Edwards et al. (LHPC), Phys. Rev. Lett. 96, 052001 (2006), hep-lat/0510062

16. A.A. Khan et al., Nucl. Phys. Proc. Suppl. 140, 408 (2005), hep-lat/0409161

17. S. Ohta, K. Orginos (RBCK), Nucl. Phys. Proc. Suppl. 140, 396 (2005), hep-lat/0411008

18. D. Dolgov et al. (LHPC), Phys. Rev. D66, 034506 (2002), hep-lat/0201021

19. D.B. Renner et al. (LHPC) (2007), arXiv:0710.1373 [hep-lat]

20. P. Hagler et al. (LHPC) (2007), arXiv:0705.4295 [hep-lat]

21. Q.M. Ohtani et al. (UKQCD) (2007), arXiv:0710.1534 [hep-lat]

22. X.S. Chen, X.F. Lu, W.M. Sun, F. Wang, T. Goldman (2007), arXiv:0709.1284 [hep-ph]

23. X.D. Ji, Phys. Rev. Lett. 78, 610 (1997), hep-ph/9603249

24. N. Mathur, S.J. Dong, K.F. Liu, L. Mankiewicz, N.C. Mukhopadhyay, Phys. Rev. D62, 114504 (2000), hep-ph/9912289

25. A. Airapetian et al. (HERMES), Phys. Rev. D75, 012007 (2007), hep-ex/0609039

26. K. Ackerstaff et al. (HERMES), Phys. Lett. B464, 123 (1999), hep-ex/9906035

27. M. Gockeler et al. (QCDSF), Phys. Rev. Lett. 98, 222001 (2007), hep-lat/0612032
28. D. Boer, P.J. Mulders, Phys. Rev. D57, 5780 (1998), hep-ph/9711485

29. C. Alexandrou et al. (2007), arXiv:0710.4621 [hep-lat]

30. I. Aznauryan, V. Burkert (2007), arXiv:0711.1120 [nucl-th]

31. H.W. Lin, R. Edwards, talk at Mini-Symposium on Baryon Resonances and Meson Production, Newport News, Virginia, October 10-13, 2007 